\title{
PREDICTING THE TOPOLOGY OF THE BENDING CORNER IN BENDING OF ULTRA HIGH STRENGTH STEELS THROUGH FINITE ELEMENT ANALYSIS
}

\author{
${ }^{1}$ Barış ÇETIN, ${ }^{2}$ Eren BILLUR, ${ }^{3}$ Besim BARANOĞLU, ${ }^{4}$ Uğur TOPTAŞ, ${ }^{4}$ Özgür ALIÇ \\ ${ }^{1}$ FNSS Defense Systems Co. Inc., Engineering and Research Department, Ankara, Turkey, \\ cetin.baris@fnss.com.tr \\ 2Billur Metal Form Ltd., Ankara, Turkey, eren@billur.com.tr \\ ${ }^{3}$ Atılım University, Metal Forming Center of Excellence Ankara, Turkey, besim.baranoglu@atilim.edu.tr \\ ${ }^{4}$ Nurol Makina ve Sanayi A.S., Ankara, Turkey, ugur.toptas@nurolmakina.com.tr
}

https://doi.org/10.37904/metal.2019.688

\begin{abstract}
In bending of plates, unlike the case of sheet metal forming, a 3-D stress state is valid. Moreover, apart from the some very specific cases, the plane strain assumption is not appropriate either. Therefore; bending of thick ultra-high strength steel (UHSS) plates is a deformation process where 3-D stress and strain states exist in general. This study basically focuses on the prediction of the bending corner topology with non-linear finite element analysis method, since the laser-cut edges of the UHSS are particularly prone to edge cracking during bending operation. Within the scope of this study, an experimental set-up is designed which consists of bending tools and a servo mechanical press. The samples were bent by means of this set-up in an air-bending operation up to $90^{\circ}$. This experimental work was followed by optical scanning measurements. And finally, the FEA results and the scanning data were compared in 3-D space. The results showed good correlation. As a future study, the 3-D strain field of the bending corner will be tried to be measured by a professional digital image correlation (DIC) system which could probably give more precise data when combined with the data from FEA.
\end{abstract}

Keywords: Metallurgy, steel, properties, applications, testing methods

\section{INTRODUCTION}

In the hull production of armored combat vehicles, there are three main steps of the process chain. These are laser cutting, bending and welding respectively. As a first step, the hull material which is generally supplied as large-sized plates are trimmed by laser cutting to the desired dimensions. Then, the plates are bent with respect to the technical drawings. For most of the cases, assuring the dimensional tolerances of the design after bending is a challenging task due to the technical difficulties such as high spring-back tendency which often necessitates some trial and error efforts in prototype production. And finally, the bent plates are transferred to welding workshop in order to obtain the hull assembly [1].

Unlike the automotive or heavy-duty machinery industry, the materials used in armored combat vehicles have larger thicknesses and dimensions. The thicknesses of the UHSS plates may reach up to $25 \mathrm{~mm}$ in certain cases so that the plane stress assumption diminishes for that type of bending operations. Moreover, for certain case here the length over thickness (L/t) ratio is not sufficiently large to ensure the plane strain condition. Therefore this contribution dealt with a specific problem which has 3-D stress and strain states by having a sample of $10 \times 200 \times 50 \mathrm{~mm}$ and a bending radius of $50 \mathrm{~mm}$. This size effect compared to sheet metal forming applications creates mainly two challenges. Firstly, the plates which have high yield strengths obviously have a high tendency of spring-back as can be seen in Figure 1. This fact generally results in some difficulties in assuring the desired dimensional tolerances defined in the technical drawings and intensively researched by many scientific groups [2-4]. 


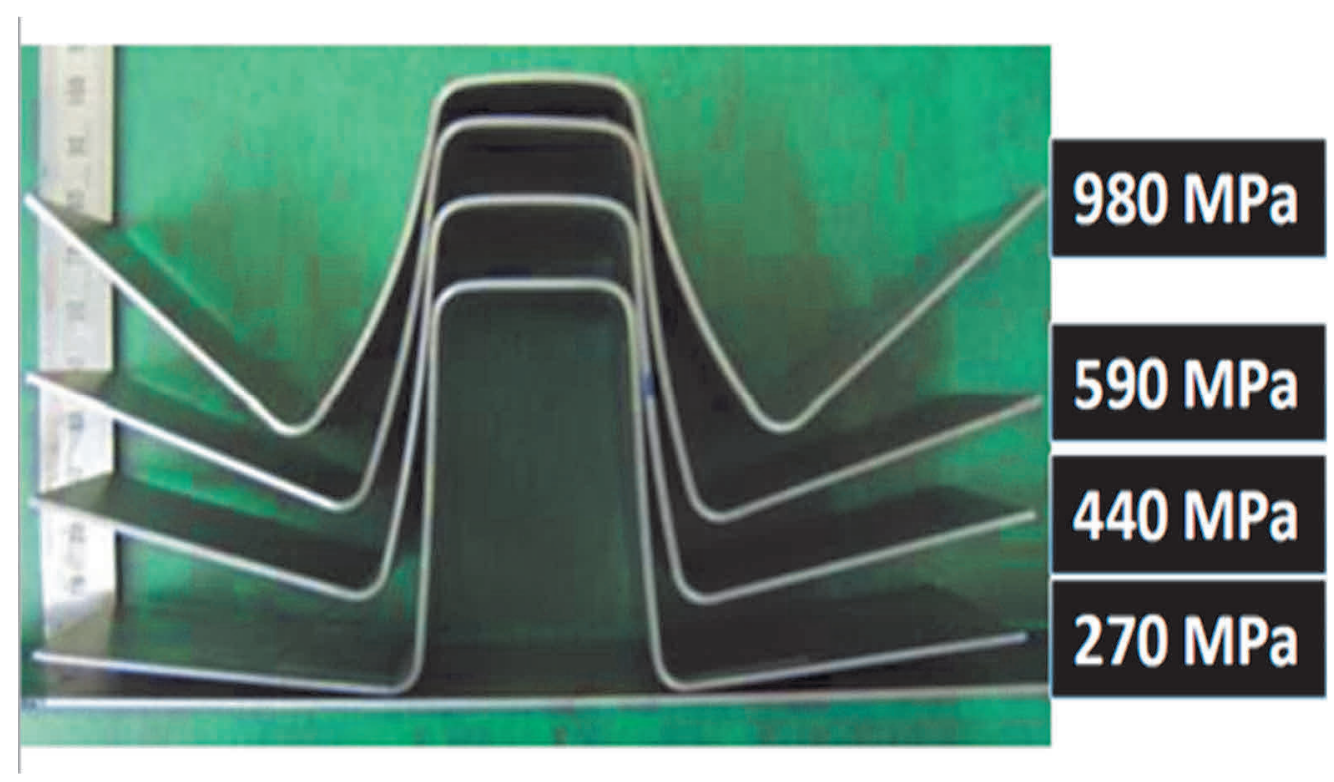

Figure 1 High spring-back tendency of HSS

Secondly, the problem has a 3-D strain state field which cannot be measured by means of 2-D digital image correlation (DIC) systems. For most of the sheet metal forming operations (plane stress cases) the computation of in plane strains and then by means of constant volume approach the determination of the equivalent strain is possible [5-7]. On the other hand, in bending of thick plates (apart from the plane strain cases where the L/t ratio is sufficiently large) more particularly in the vicinity of the bending corner region the plastic deformation is fairly localized and the gradient of plastic strain might be reasonably high. This fact also provokes the fracture of the material (see Figure 2) because of which the topology of the bending corner plays an important role in designing and the simulation of the bending process. Furthermore, this fact also eliminates the possible implementations of mean in-plane strain calculations. Therefore, the whole topology should be obtained to perform scientifically meaningful computations and comments of the 3-D strain field. After having precisely obtaining the spatial equivalent strain data then one can predict or comment on the possible fracture tendency in the bending corner.

In that context, this contribution deals with the prediction of the bending corner topology in bending of thick UHSS plates through FEA simulations. This basic but essential step should be performed successfully before having determining the direct components of a strain tensor. Therefore, within the scope this study the FEA prediction of the topology of bending corner is compared by the optical scanning data. For that purpose, FEA software package of MSC Marc and Alicona Infinite-Focus optical measurement system is used. As far as the knowledge of the authors there does not exist any similar studies in the open literature concerning the present specific subjet. One of the distinguishing aspect of this study is also creating a special MatLab script which is capable of importing stl data of Alicona system and node coordinates of the FEA. The script than could also conduct a point to point comparison and plot the $\Delta Z$ values as a spatial distribution in $X Y$ plane.

\section{FINITE ELEMENT ANALYSIS (FEA) STUDIES}

FEA analysis were performed in commercial MSC Marc software. The bending operation is modelled with an elasto-plastic isotropic hardening material model with Von Mises's yield criterion. The bending tools were also assumed to be rigid and a combined friction model (Coulomb + Viscous) is integrated to the simulations. And finally regarding the numerical computations, the implicit time integration method is engaged. The simulation environment and some results regarding the equivalent plastic strain predictions are illustrated in Figure 2. 
The studied material in this manuscript is a UHSS plate whose yield strength is nearly $1250 \mathrm{MPa}$ and ultimate tensile strength (UTS) is $1750 \mathrm{MPa}$ conform to Mil-Dtl-46100 standard.

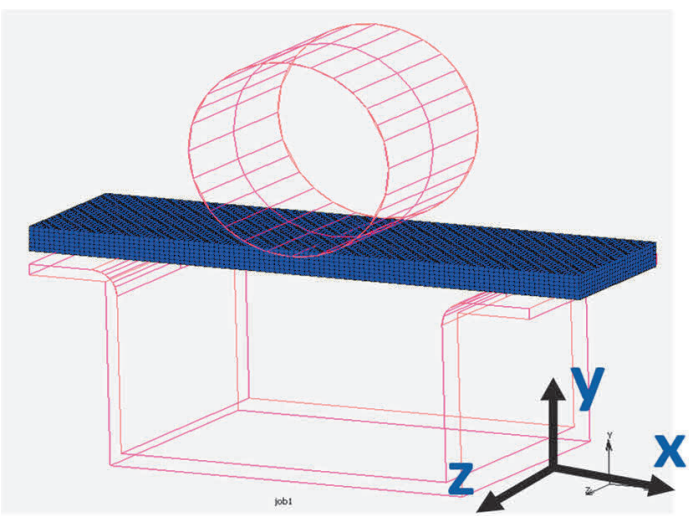

Figure 2a Simulation model in MSM Marc
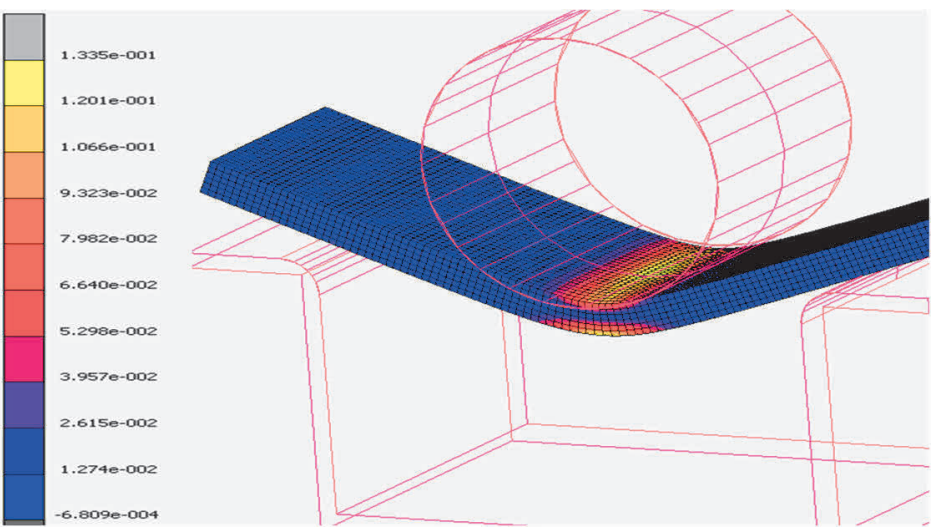

Figure 2b Equivalent plastic strains as color map

During FEA analysis, some sensitivity analysis is performed regarding the element size, element type and the coefficient of friction. The comparative results were illustrated in Section 4. As a final step of FEA analysis, the node coordinates are extracted from the software to construct the surface topology. To perform this task, a specific script is created in MatLab environment (see Figure 4).

\section{EXPERIMENTAL STUDIES}

Air bending experiments were performed using a 80 ton servo-mechanical press which is located at Atılım University Metal Forming Center of Excellence (MFCE). The press has a knuckle-joint mechanism and bending was done at $100 \%$ speed profile (i.e., servo functions were not utilized). The bent samples were then examined by Alicona Infinite-Focus optical measurement system. The experimental set-ups are illustrated in Figure 3a and $3 \mathbf{b}$.

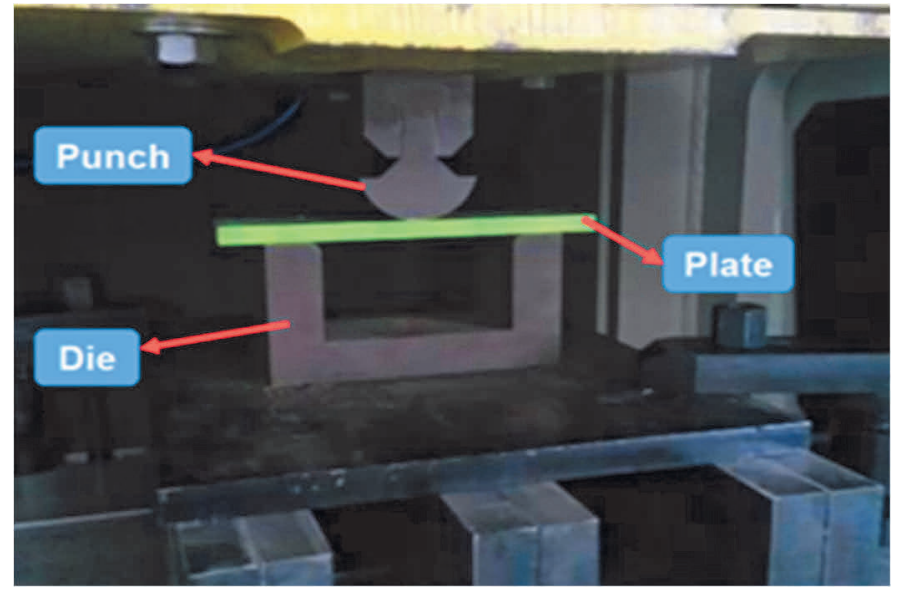

Figure 3a Air bending set-up

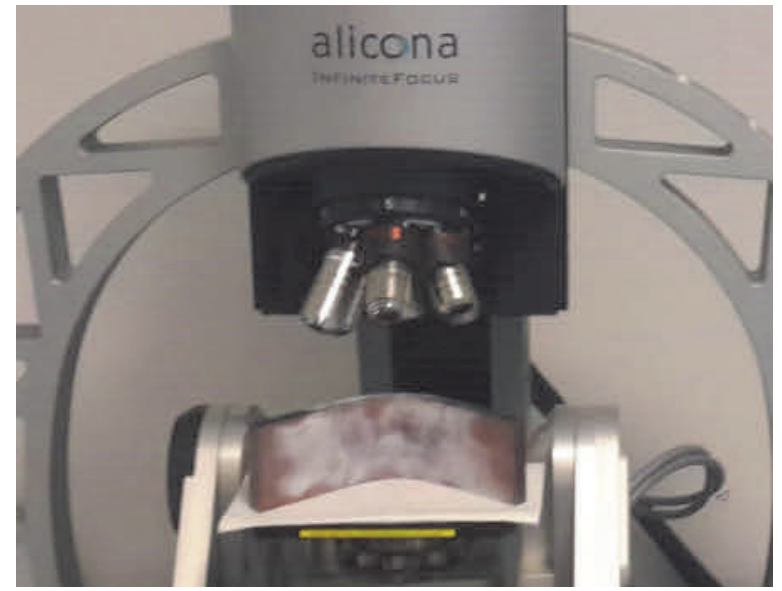

Figure 3b Optical scanning set-up

During the optical scanning operation the $2.5 \mathrm{X}$ lens in used with the exposure time of 600 microseconds. In that configuration, the vertical resolution of 550 nanometer and the lateral resolution of 41 micrometer are provided by the Alicona system. Then, by means of the script which is created by our research group, stl data is plotted as a 3D surface in MatLab environment as in Figure 4. 


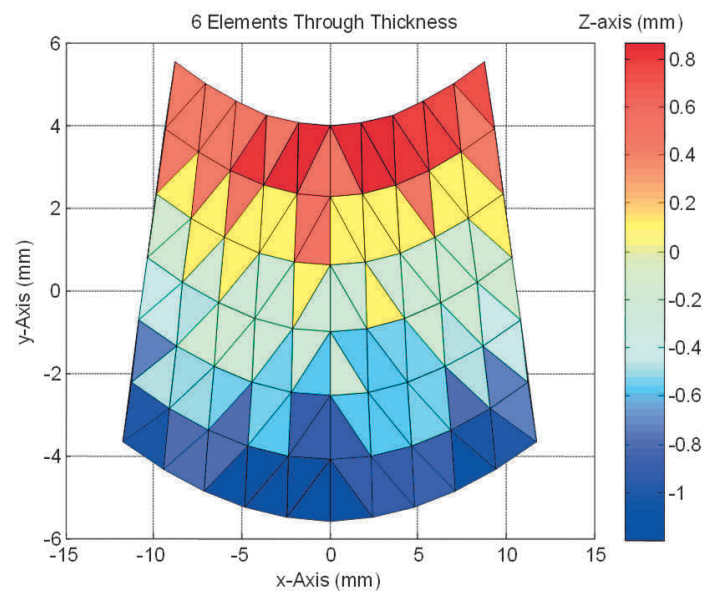

Figure 4a Simulation result for 6 elements in thickness direction

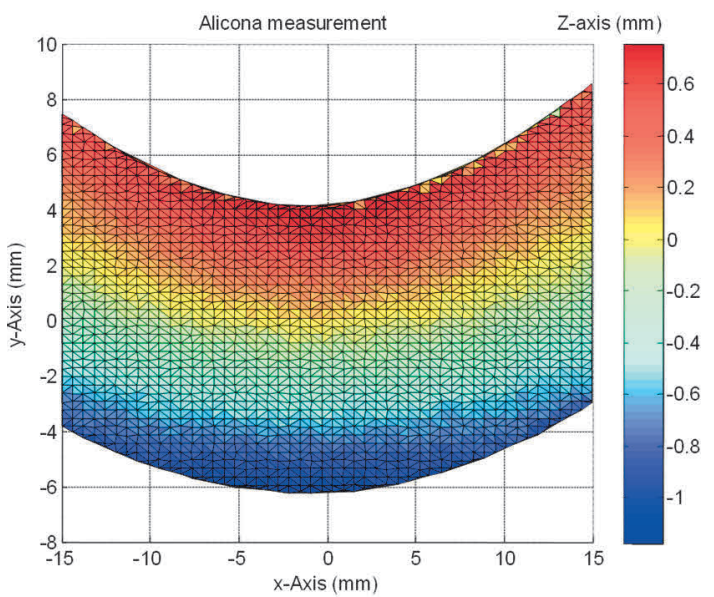

Figure 4b Resulting surface data of optical scanning

\section{RESULTS AND DISCUSSION}

The most critic region where the plastic strain is maximum is the inner centroid of the bent specimen which is in contact with the punch with a radius of $40 \mathrm{~mm}$. Therefore, it is convenient to observe any effect of the coefficient of friction (cof) in the bending corner topology. Figure 5.a illustrates the simulation results of the having cof of $0.08,0.10$ and 0.15 for the centroid nodes and 5.b shows the same results as the average value and the dispersion. This comparative study is performed for the condition of six element in the thickness direction. The results revealed that up to some extend cof have some effect ( $\% 5$ max) on the topology. Moreover the lower cof values stimulate the local plastic deformation, i.e. the change in Z-direction increases, as expected.

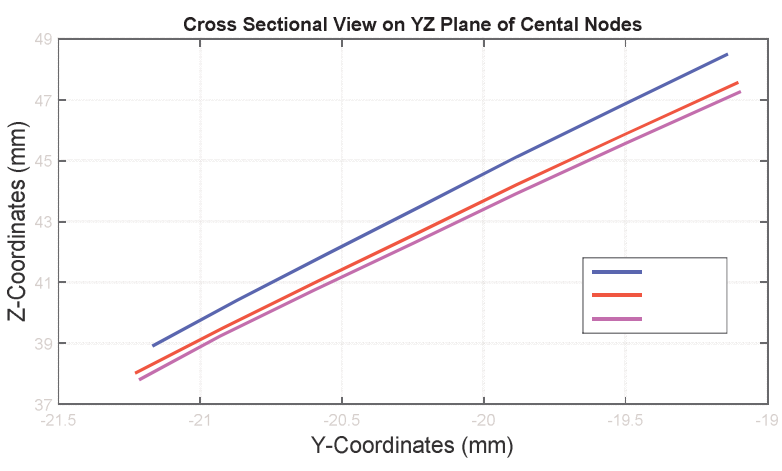

Figure 5a $Y$ and $Z$ coordinates of the central nodes for specific cof

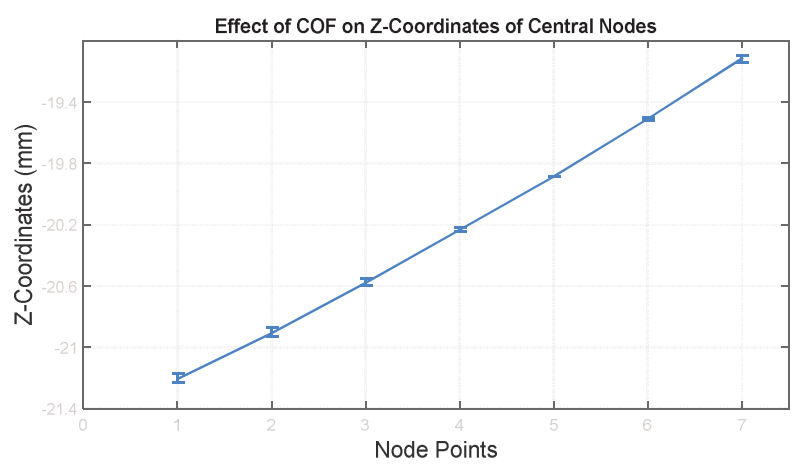

Figure 5b Error bar graph of the Z-coordinates regarding three specific cof

The comparison of linear elements (Hex-8) and the quadratic elements (Hex-20) is shown in Figure 6.b. The mean difference (point-wise average) between linear and quadratic elements is lower than 0.51 micrometers whereas the computation time is increased as 80 times. Therefore, it is concluded to use linear (Hex-8) elements in our simulations as an optimization. Figure 6.a also gives the results of the simulation whose configuration has 10 through thickness elements. When Figure 6.a is investigated together with Figure 4.a, it would be concluded that both simulations have very similar predictions. As in the case of element formulation, the element quantity in the thickness direction has not any reasonable effect. The mean difference is still less than 1.30 micrometers. To conclude, it can be stated that in both sensitivity analysis cases, the difference is less than $0.10 \%$. 


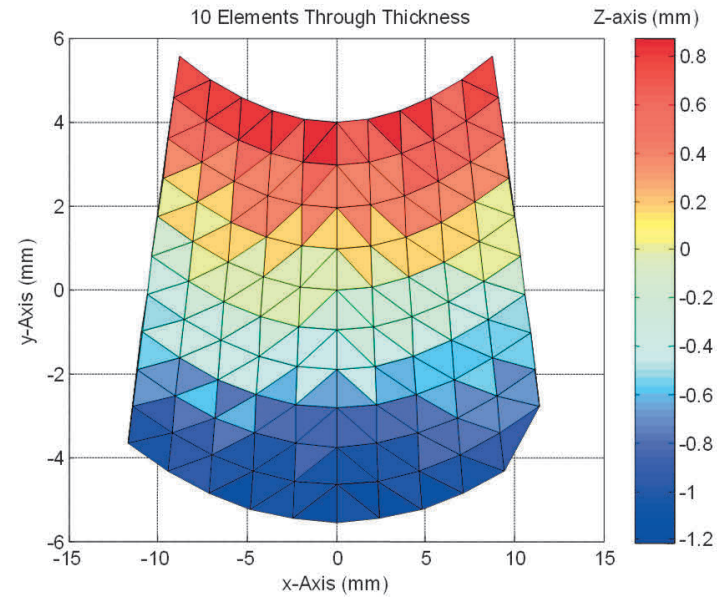

Figure 6 a Simulation result for 10 elements in thickness direction

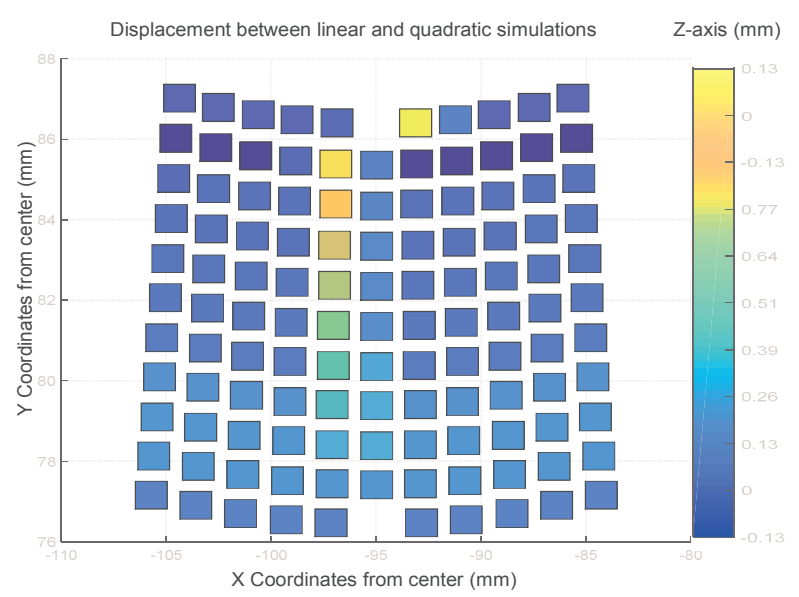

Figure 6b Comparison of linear and quadratic element formulation

And finally, the comparison of the simulation result with the optical scanning data is illustrated in Figure 7. As it can be observed the mean difference (point-wise) between the two surfaces is $0.10643 \mathrm{~mm}$ which can be converted as $6.2 \%$ variation with respect to the max $\Delta Z$ value. This precision amount is considered as acceptable by our research group in case of industrial applications.

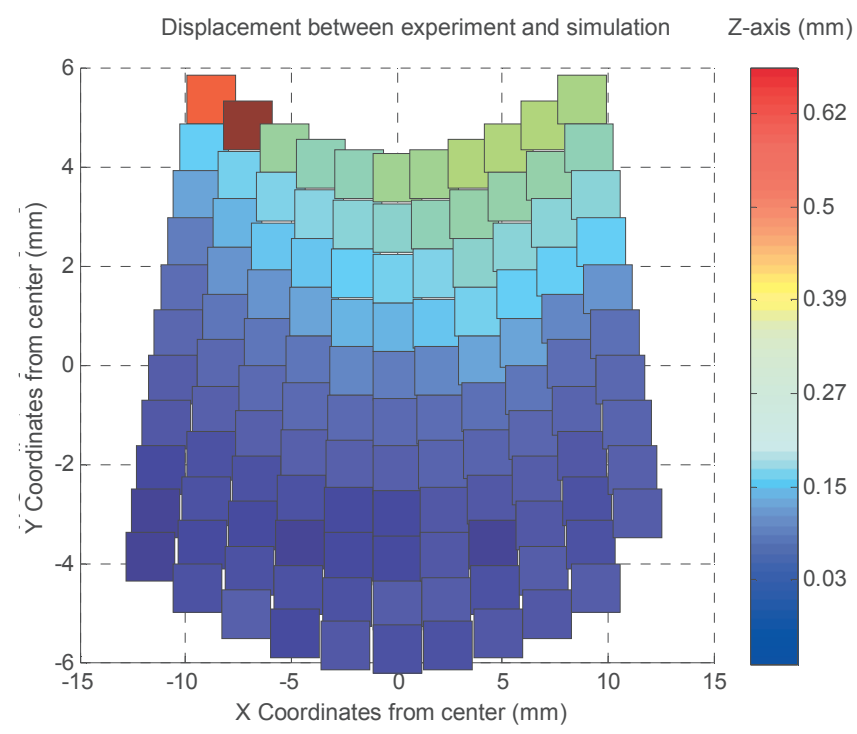

Figure 7 Comparison of simulation and experimental data

\section{CONCLUSION}

The following outputs could be stated as concluding remarks which were obtained from the present work:

- $\quad$ For the case of the thick plate bending (apart from the plane strain cases) the bending corner has a 3D surface topology like free surface geometry which eliminates any mean equivalent in-plane strain assumption. When the extreme points is concerned the difference in the Z-direction is nearly $1.70 \mathrm{~mm}$. This fact implies that the spatial equivalent strain have to be computed with the six individual component of the strain tensor. 
- In that sense, owing to the difficulties in having sophisticated digital image correlation (DIC) systems in the workshop and recording the strain data, FEA could be a powerful alternative instead.

- It is observed that the element formulation in FEA has a negligible effect on the bending corner topology when industrial application is concerned. Moreover, usage of the quadratic elements (Hex-20 in MSC Marc) increased the computation time enormously.

- As expected the coefficient of friction (cof) has an unneglectable effect. Regarding the extreme points, change of cof to 0.08 to 0.15 results in nearly $4.60 \%$ dimensional change.

- $\quad$ After having performed the necessary sensitivity analysis on FEA, the bending corner topology can be predicated with a reasonable amount of precision. Then, the equivalent plastic strain can also readily be used in failure prediction. If the damage criteria is valid and integrated into the simulation, the possible cracking phenomena on the bending corner zone may also be predicted by FEA software.

- This manuscript discussed the bending topology prediction in bending of thick UHSS plates and proposed a method on FEA. The failure prediction on the subject will be treated as a future work by our research group in which the preliminary efforts have been already initiated.

\section{ACKNOWLEDGEMENTS}

This contribution presents a certain portion of an internally-funded R\&D project of FNSS and Nurol Makina companies. Therefore, the authors would like to thank to managerial boards of FNSS Defense Systems Co. Inc. and Nurol Makina Sanayi A.S. for their financial supports. Thank are also extended to MFCE for the use of their facilities.

\section{REFERENCES}

[1] CETIN, Baris. Master Thesis. Atılım University, Graduate School of Natural and Applied Science. 2018

[2] ZHANG, M., ZHANG, J., NING, Y.X., WANG, T. and WAN, Z. Springback Behavior of Advanced High Strength Steel CP800. Metallic Materials and Manufacturing Technology. 2013. vol. 820, no. 2, pp. 45-49.

[3] YANG, X., CHOI, C., SEVER, N.K. and ALTAN, T. Prediction of Springback in Air-bending of AHSS (DP780) Considering Young's Modulus Variation and with A Piecewise Hardening Function. International Journal of Mechanical Sciences. 2016. vol. 105, pp. 266-272.

[4] SEO, K.Y., KIM, J.H., LEE, H.S., KIM, J. and KIM, B.M. Effect of Constitutive Equations on Springback Prediction Accuracy in the Trip1180 Cold Stamping. Metals. 2018. vol. 8, pp. 18-35.

[5] ABGHANI, A.F., ALI, M.B., DHARMALINGAM, S. and MAHMUD, J. Digital Image Correlation Technique in Measuring Strain Using Open-source Platform NCorr. Journal of Advanced Research in Applied Mechanics. 2016. vol. 26, no. 1, pp. 10-21.

[6] SEYMEN, Y., GULER, B. and EFE, M. Large Strain and Small-Scale Biaxial Testing of Sheet Metals. Experimental Mechanics. 2016. vol. 20, no. 2, pp. 102-112.

[7] EFE, Mert. Strain Localization Behavior of Cold-Rolled Deep-Drawing Steels. Celal Bayar University Journal of Science. 2019. vol.16, no.1, pp. 81-86. 